\title{
Premature Ejaculation and Erectile Dysfunction in Iranian Prostate Cancer Patients
}

\author{
Chung-Ying Lin ${ }^{1}$, Andrea Burri², Amir H Pakpour ${ }^{3 *}$
}

\begin{abstract}
Background: To investigate the prevalence of premature ejaculation (PE) and erectile dysfunction (ED) in a sample of patients with prostate cancer and to determine the utility of the previously suggested cutoffs of the Premature Ejaculation Diagnostic Tool (PEDT) for the diagnosis of PE and that of International Index of Erectile Function (IIEF-5) for ED. Materials and Methods: A total of 1,202 men with prostate cancer were invited from urology clinics at the universities of Iran, Tehran, Qazvin, Ahvaz, Guilan and Tabriz. Clinical characteristics were collected through medical records. $P E$ and $E D$ diagnoses were made by trained urologists. In addition to the clinical diagnoses, PE and ED were measured through self-report using the PEDT and the IIEF-5. Questionnaire cutoff scores were determined using receiver operating characteristic (ROC) curves and confirmed by predictive ability using logistic regression. Results: The prevalence of PE was 63.7\% and that of ED was 66.2\%. Prevalences of PE decreased and that of ED increased with advanced TNM stages. According to ROC, the suggested cutoff for the PEDT to diagnose a PE was $\geq 11$ (sensitivity=0.988, 1 -specificity=0.084, and predictive ability $=0.914$ ) and

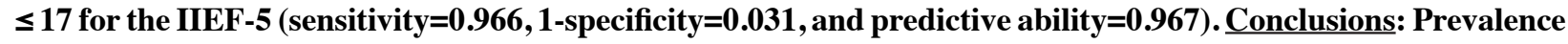
of sexual problems was high in prostate cancer patients in Iran, therefore oncologists should take into account these potential problems when deciding on treatment modalities.
\end{abstract}

Keywords: Erectile dysfunction - prostate cancer - premature ejaculation - prevalence - PEDT - IIEF-5.

Asian Pac J Cancer Prev, 17 (4), 1961-1966

\section{Introduction}

Premature ejaculation (PE) and erectile dysfunction (ED) are the two most common sexual problems in male population with reported prevalences of up to $38 \%$ (Spector and Carey, 2009) for PE and up to 52\% for ED (Rosen et al., 1999). Prevalences tend to be higher in certain clinical populations, such as for example men suffering from short frenulum (Gallo et al., 2010), and those who had worse control over ejaculation (Giuliano et al., 2014; Gagnon and Polverejan,2008).This may also be true for prostate cancer patients who have reported problems relating to erectile and ejaculatory function caused by certain treatments (e.g., radiotherapy) (Singer et al., 1991; Benson et al.,2012) and have shown to accept at least a $10 \%$ decrement in survival for a treatment that grants a better chance of preserving their erectile function (Incrocci et al., 2002); Therefore, assessing the prevalence of $\mathrm{PE}$ and $\mathrm{ED}$ in prostate cancer patients is critical in the cancer epidemiology.

Although the diagnosis of PE and ED is often made by urologists, the trend of applying self-report measures to assess ejaculatory and erectile problems is growing - not only for research purposes but also in clinical settings (Symonds et al., 2007; Chen et al., 2014). The benefits of using such measures are obvious as they offer the possibility to gather a lot of data by the simple and quick administration of short questionnaires to evaluate the sexual function in large population samples. Two such validated and very widely used questionnaires are the Premature Ejaculation Diagnostic Tool (PEDT) for the assessment of ejaculatiory function (Symonds et al., 2007) and the International Index of Erectile Function (IIEF-5) for the assessment of erectile function (Rosen et al., 1999) - both of which consist of 5 items only. Additionally, for both questionnaires cut-off scores have been proposed in the past that can be for the clinical diagnosis of PE and ED, respectively (Rosen et al., 1999; Symonds et al., 2007). Specifically, Rosen et al.(1999) examine the sensitivity of the IIEF-5 using a ED sample and an apparently healthy sample from the UK and the US, and suggest the IIEF-5 score $<22$ as having ED. Symonds et al. (2007) examine the sensitivity of the PEDT using a PE sample and an apparently healthy sample all from the US, and suggest the PEDT score $>8$ as having PE. However, whether these previously proposed cutoff scores of the PEDT and the

${ }^{1}$ Department of Public Health, College of Medicine, National Cheng Kung University, Tainan, Taiwan as Department of Rehabilitation Sciences, Faculty of Health and Social Sciences, The Hong Kong Polytechnic University, Hung Hom, Hong Kong ${ }^{2}$ Department of Psychology, University of Zurich, Zurich, Switzerland; ${ }^{3}$ Social Determinants of Health Research Center (SDH), Qazvin University of Medical Sciences, Qazvin,Iran *For correspondence:Pakpour_Amir@yahoo.com, 
IIEF-5 can be extrapolated to prostate cancer patients remains unclear.

Given the growing use of assessing sexual function using self-report measures such as the PEDT and IIEF-5 not only in the general population but also in patient cohorts, such as men suffering from prostate cancer, (Bianco, et al., 2009; Choo et al., 2010; Ong et al., 2015; Saitz et al., 2013), clinicians, especially oncologists, should know how to adopt the two useful instruments in their clinical decision making. It is therefore crucial to present specific cutoffs for the PEDT and the IIEF-5 to be able to validly diagnose sexual problems in cancer patient samples in order to foster research on the aetiological factors underlying the development of such problems in these cohorts.

According to this, the purposes of this study were (1) to explore the prevalence of PE and ED in a sample of Iranian men suffering from prostate cancer; (2) to determine the cutoffs of the PEDT and the IIEF-5 for the diagnosis of sexual problems in prostate cancer patients; (3) to investigate potential factors that impact on the sexual function of prostate cancer patients.

\section{Materials and Methods}

\section{Participants}

Data collection was conducted from March 2014 to August 2015. Participants were 1202 men with a diagnosis of prostate cancer and who had been referred to a Urology Clinic for treatment purposes. All patients were randomly selected from Urology Clinics at the universities of Iran, Tehran, Qazvin, Ahvaz, Guilan and Tabriz. Potential participants had to be at least 18 years old, be in a stable sexual relationship with a female partner for at least 6 months, and had to consent to participate in the study. Patients were excluded if they were unable to understand Persian, refused to participate, or if they were too ill or cognitively impaired to give consent. Patient's cognitive functioning was evaluated using the Mini-Mental State Examination (MMSE). A score of 23 or less was considered as evidence for cognitive impairment. Of the 1202 approached patients, 81 patients were not eligible to be included in the study, and 63 refused to participate, resulting in a final sample of $\mathrm{N}=1058$. Additionally, four participants had missing information regarding their PEDT and IIEF-5 scores. Finally there were 1054 participants for data analysis, and their demographics were shown in Table 1. All research tools including the PEDT, the IIEF5, and the MMSE were completed by the patients at the Urology Clinic during their visits.

Clinical data for each patient were retrieved from subject's medical record. Moreover, PE and ED were assessed by two trained urologists according to the Diagnostic and Statistical Manual of Mental Disorders, Fifth Edition (DSM-5) criteria (2013).

The Ethics Committee of Qazvin University of Medical Sciences approved this study before recruitment, and all participants provided written informed consent.

\section{Instruments}

Premature Ejaculation Diagnostic Tool: THE PEDT is a 5-item self-report used to evaluate men's ejaculatory function based on the DSM-IV-TR criteria for PE (2000). The developers found the PEDT to have satisfactory psychometric properties, including good internal consistency (Cronbach's $\alpha=0.71$ ), test-retest reliability $(\mathrm{r}=0.73)$, and known-group validity (significantly distinguished time-defined PE and non-PE groups) (Symonds et al., 2007). In addition, the following cutoffs for PE have been suggested: $\geq 11$ suggests a diagnosis of PE, 9 and 10 of probable PE, and $\leq 8$ of non-PE (Symonds et al., 2007). Using the cutoff suggested by Raymonds, (Symonds et al., 2007) a Persian questionnaire version exists that has also shown to have excellent psychometric properties with an internal consistency of 0.89 and good valdity for an Irania sample, including a PE sample and an apprentaly healthy control (Pakpour et al., 2014a).

International Index of Erectile Function (IIEF-5): The IIEF-5, a short version of the longer 15-item IIEF, was developed for the quick evaluation of men's erectile function (Rosen et al., 1999). In a sample consists of ED patients and healthy men, the following cutoffs for ED were defined: $\leq 7$ suggests severe ED, 8-11 moderate ED, 12-16 mild to moderate ED, 17-21 mild ED, and $\geq 22$ non-ED (Rosen et al., 1999).The 15-item IIEF has also been translated into Persian language for the use in Iranian populations with sound psychometric properties (Pakpour et al., 2014b).

\section{Statistical analysis}

All analyses were done using SPSS 17.0 (SPSS Inc., Chicago, IL). Prevalences were calculated using the number (proportion) of participants suffering of PE and ED, respectively. Prevalances were assessed for all participants, and according to the different stages of cancer progression (TNM). Receiver operating characteristics (ROC) curves were created in which the sensitivity (aka true-positive rate) and the 1-specificity (aka falsepositive rate) were paired across all potential cutoffs that distinguished participants with a sexual problem (PE or ED) from cancer patients without (Rosen et al., 1999). The urologists' evaluation was used as the gold standard to test the usefulness of the PEDT and IIEF-5 scores to distinguish sexually healthy from dysfunctional patients. Area under the ROC curve (AUC) was used to determine whether the PEDT and IIEF-5 scores are valid, with an AUC of $>0.75$ suggesting clinical usefulness and $>0.97$ very high clinical value (Fan et al., 2006). In addition to the sensitivity and 1-specificity profile, predictive ability was also computed using logistic regressions to examine the accuracies of candidate cutoffs for the PEDT (candidate cutoffs ranging from $\geq 10$ to $\geq 14$ ) and for the IIEF-5 (candidate cutoffs ranging from $\leq 13$ to $\leq 19$ ). After analyzing the ROC curves for the all participants, the sample was divided into help-seeking and non-seeking individuals to reanalyze the ROC curves.

Multiple linear regressions and ordinal logistic regressions were conducted to determine potential predictors of PE and ED. The same independent variables were used for both linear and ordinal logistic regressions including are age, time after diagnosis, years of education, 
DOI:http://dx.doi.org/10.7314/APJCP.2016.17.4.1961 Premature Ejaculation and Erectile Dysfunction in Iranian Prostate Cancer Patients

Table 1. Participant Characteristics and Scores of Premature Ejaculation Diagnostic Tool (PEDT) and International Index of Erectile Function (IIEF-5)

\begin{tabular}{|c|c|c|c|c|}
\hline Basic characteristics: Mean \pm SD & $\begin{array}{c}\mathrm{PE} \\
(\mathrm{n}=674)\end{array}$ & $\begin{array}{l}\text { Non-PE } \\
(\mathrm{n}=380)\end{array}$ & $\begin{array}{c}\text { ED } \\
(n=698)\end{array}$ & $\begin{array}{l}\text { Non-ED } \\
(\mathrm{n}=356)\end{array}$ \\
\hline Age (years) & $64.39 \pm 6.85$ & $63.56 \pm 6.73$ & $63.97 \pm 6.57$ & $64.33 \pm 7.27$ \\
\hline Years of educationa & $5.12 \pm 1.28$ & $5.08 \pm 1.19$ & $5.03 \pm 1.22$ & $5.27 \pm 1.29$ \\
\hline Duration-after-diagnosis (years) & $6.20 \pm 3.55$ & $6.01 \pm 3.32$ & $6.17 \pm 3.45$ & $6.06 \pm 3.52$ \\
\hline Body mass index $(\mathrm{kg} / \mathrm{m} 2)$ & $24.52 \pm 4.33$ & $24.04 \pm 4.63$ & $24.03 \pm 4.64$ & $24.98 \pm 3.95$ \\
\hline \multicolumn{5}{|l|}{ Clinical characteristics: $\mathrm{n}(\%)$} \\
\hline \multicolumn{5}{|l|}{ Gleason grade at diagnosis } \\
\hline Low & $159(23.6)$ & $97(25.5)$ & $169(24.2)$ & $87(24.4)$ \\
\hline Medium & $282(41.8)$ & $156(41.1)$ & $296(42.4)$ & $142(39.9)$ \\
\hline High & $156(23.1)$ & $92(24.2)$ & $156(22.3)$ & $92(25.8)$ \\
\hline Unknown & $77(11.4)$ & $35(9.2)$ & $77(11.0)$ & $35(9.8)$ \\
\hline \multicolumn{5}{|l|}{ Stage at diagnosis } \\
\hline 1 & $126(18.7)$ & $40(10.5)$ & $102(14.6)$ & $64(18.0)$ \\
\hline 2 & $302(44.8)$ & $159(41.8)$ & $282(40.4)$ & $179(50.3)$ \\
\hline 3 & $183(27.2)$ & $139(36.6)$ & $231(33.1)$ & $91(25.6)$ \\
\hline Unknown & $63(9.3)$ & $42(11.1)$ & $83(11.9)$ & $22(6.2)$ \\
\hline \multicolumn{5}{|l|}{ PEDT and IIEF-5 scores: Mean \pm SD } \\
\hline PEDT item1 & $3.01 \pm 1.04$ & $1.47 \pm 1.30$ & $2.42 \pm 1.44$ & $2.52 \pm 1.19$ \\
\hline PEDT item2 & $2.98 \pm 1.08$ & $1.20 \pm 1.30$ & $2.21 \pm 1.51$ & $2.60 \pm 1.29$ \\
\hline PEDT item 3 & $3.57 \pm 0.87$ & $2.06 \pm 1.44$ & $2.84 \pm 1.39$ & $3.39 \pm 1.11$ \\
\hline PEDT item4 & $3.65 \pm 0.74$ & $1.58 \pm 1.43$ & $2.70 \pm 1.51$ & $3.30 \pm 1.20$ \\
\hline PEDT item5 & $3.33 \pm 1.10$ & $1.17 \pm 1.38$ & $2.39 \pm 1.62$ & $2.87 \pm 1.48$ \\
\hline PEDT total score & $16.54 \pm 2.92$ & $7.47 \pm 4.57$ & $12.55 \pm 5.87$ & $14.68 \pm 4.90$ \\
\hline IIEF-5 item 1 & $3.12 \pm 1.57$ & $3.06 \pm 1.51$ & $2.49 \pm 1.48$ & $4.30 \pm 0.81$ \\
\hline IIEF-5 item 2 & $3.01 \pm 1.77$ & $2.79 \pm 1.67$ & $2.12 \pm 1.52$ & $4.51 \pm 0.76$ \\
\hline IIEF-5 item3 & $2.62 \pm 1.72$ & $2.22 \pm 1.53$ & $1.61 \pm 1.16$ & $4.17 \pm 1.09$ \\
\hline IIEF-5 item4 & $2.36 \pm 1.73$ & $2.35 \pm 1.65$ & $1.68 \pm 1.26$ & $3.70 \pm 1.65$ \\
\hline IIEF-5 item5 & $2.61 \pm 1.73$ & $2.29 \pm 1.53$ & $1.63 \pm 1.20$ & $4.19 \pm 1.04$ \\
\hline IIEF total score & $13.72 \pm 7.15$ & $12.71 \pm 6.18$ & $9.52 \pm 4.74$ & $20.87 \pm 2.99$ \\
\hline
\end{tabular}

$\mathrm{PE}=$ premature ejaculation; $\mathrm{ED}=$ erectile dysfunction

job status (reference was no job), smoking status (reference was not smoking), seeking help (reference was not seeking for a job), body mass index (BMI), Gleason grades at diagnosis (reference was high), TNM staging at diagnosis (reference as stage 3 ), and the IIEF-5 (for ED) or PEDT (for PE) scores. The dependent variables were the PEDT and IIEF-5 total scores for the linear regressions, and different groups based on levels of PEDT (no PE: $\leq 8$, probable PE: 9-10, and PE: $\geq 11$ ) and IIEF-5 (absent: $\geq$ 22, mild: $17-21$, mild-moderate: $12-16$, moderate: $8-11$, and severe: 57 ) for ordinal logistic regressions. $\beta$ s were presented for linear regressions, and odds ratio (OR) with $95 \%$ confidence interval (CI) for ordinal logistic regressions.

\section{Results}

The mean age of the participants was 64.07 years (SD 6.84). Duration-after-diagnosis was 6.14 (3.47) years and BMI was 24.34 (4.44). Based on Gleason grading the severity of the prostate cancer at first diagnosis was classified as low in 259 individuals (24.5\%), medium in 438 individuals (41.4\%), high in 248 individuals (23.4\%), and unknown in 113 individuals (10.7\%). In addition and according to TNM staging system,166 patients $(15.7 \%)$ were diagnosed as stage $1,462(43.7 \%)$ as stage 2,325 $(30.7 \%)$ as stage 3 , and $105(9.9 \%)$ as unknown.

The prevalence of PE and ED varied based on different diagnostic methods. According to the urologists'
Table 2. Prevalence of PE and ED Stratified by Gleason Grade and Stage at Diagnosis

\begin{tabular}{llcc}
\hline & \multicolumn{2}{c}{$\mathrm{n}(\%)$} \\
\cline { 2 - 3 } & \multicolumn{2}{c}{ PE } & ED \\
\hline Based on clinical evaluation & & \\
All patients & & $674(63.7 \%)$ & $698(66.2 \%)$ \\
Gleason grade $^{\text {a }}$ & Low & $159(62.1 \%)$ & $169(66.0 \%)$ \\
& Medium & $282(64.4 \%)$ & $296(67.6 \%)$ \\
Stage $^{a} 1$ & High & $156(62.9 \%)$ & $156(62.9 \%)$ \\
2 & & $169(66.0 \%)$ & $102(61.4 \%)$ \\
& & $296(67.6 \%)$ & $282(61.2 \%)$ \\
& & $156(62.9 \%)$ & $231(71.7 \%)$
\end{tabular}

Based on PEDT (cutoff: $\geq 11$ ) or IIEF-5 scores (cutoff: $\leq 7$ )

\begin{tabular}{|c|c|c|c|}
\hline \multirow{2}{*}{$\begin{array}{l}\text { All patients } \\
\text { Gleason grade }\end{array}$} & & $749(71.1 \%)$ & $331(31.4 \%)$ \\
\hline & Low & $181(70.7 \%)$ & $80(31.3 \%)$ \\
\hline & Medium & $311(71.0 \%)$ & $142(32.4 \%)$ \\
\hline & High & $176(71.0 \%)$ & $76(30.6 \%)$ \\
\hline Stage $^{\mathrm{a}} 1$ & & $143(86.1 \%)$ & $59(35.5 \%)$ \\
\hline 2 & & $332(72.0 \%)$ & $129(28.0 \%)$ \\
\hline 3 & & $212(65.8 \%)$ & $106(32.9 \%)$ \\
\hline
\end{tabular}

Based on PEDT (cutoff: $\geq 9$ ) or IIEF-5 scores (cutoff: $\leq 22$ )

\begin{tabular}{|c|c|c|c|c|}
\hline \multirow{2}{*}{\multicolumn{2}{|c|}{$\begin{array}{l}\text { All patients } \\
\text { Gleason grade }\end{array}$}} & & $829(78.7 \%)$ & $907(86.1 \%)$ \\
\hline & & Low & $203(79.3 \%)$ & $225(87.9 \%)$ \\
\hline & & Medium & $343(78.3 \%)$ & $376(85.8 \%)$ \\
\hline & & High & $194(78.3 \%)$ & $216(87.1 \%)$ \\
\hline Stage $^{a}$ & 1 & & $146(87.9 \%)$ & $139(83.7 \%)$ \\
\hline & 2 & & $373(80.9 \%)$ & $390(84.6 \%)$ \\
\hline & 3 & & $239(74.2 \%)$ & $286(88.8 \%)$ \\
\hline
\end{tabular}

aPatients with unknown Gleason grade and/or unknown stage were omitted in the prevalence reports 
Table 3. Diagnostic Statistics, Sensitivity, 1-Specificity, and Predictive Ability for Premature Ejaculation Diagnostic Tool (PEDT) and International Index of Erectile Function (IIEF-5)

\begin{tabular}{|c|c|c|c|c|}
\hline $\begin{array}{c}\text { Measures } \\
\text { Candidate cutoff }\end{array}$ & AUC $(95 \% \mathrm{CI})$ & Sensitivity & 1-Specificity & Predictive ability \\
\hline PEDT & $0.944(0.925-0.963)$ & -- & -- & -- \\
\hline$\geq 10$ & - & 0.988 & 0.218 & 0.873 \\
\hline$\geq 11$ & -- & 0.988 & 0.084 & 0.914 \\
\hline$\geq 12$ & -- & 0.915 & 0.079 & 0.962 \\
\hline$\geq 13$ & -- & 0.837 & 0.071 & 0.917 \\
\hline$\geq 14$ & -- & 0.754 & 0.066 & 0.87 \\
\hline IIEF-5 & $0.969(0.957-0.981)$ & -- & -- & -- \\
\hline$\leq 13$ & -- & 0.762 & 0.022 & 0.835 \\
\hline$\leq 14$ & -- & 0.798 & 0.022 & 0.859 \\
\hline$\leq 15$ & -- & 0.84 & 0.028 & 0.884 \\
\hline$\leq 16$ & -- & 0.904 & 0.028 & 0.927 \\
\hline$\leq 17$ & -- & 0.966 & 0.031 & 0.967 \\
\hline$\leq 18$ & -- & 0.968 & 0.152 & 0.928 \\
\hline$\leq 19$ & -- & 0.973 & 0.284 & 0.886 \\
\hline
\end{tabular}

$\mathrm{AUC}=$ area under the receiver operating characteristic curve (ROC); $\mathrm{CI}=$ confidence interval

Table 4. Predictors on Premature Ejaculation Diagnostic Tool (PEDT) and International Index of Erectile Function (IIEF-5) scores

\begin{tabular}{|c|c|c|c|c|c|c|c|c|}
\hline \multirow[b]{3}{*}{ Predictors } & \multicolumn{4}{|c|}{ Linear regression } & \multicolumn{4}{|c|}{ Ordinal logistic regression } \\
\hline & \multicolumn{2}{|l|}{ PEDT } & \multicolumn{2}{|l|}{ IIEF-5 } & \multicolumn{2}{|r|}{ PEDT } & \multicolumn{2}{|r|}{ IIEF-5 } \\
\hline & $\beta(\mathrm{SE})$ & $\mathrm{p}$ & $\beta(\mathrm{SE})$ & $p$ & OR & $(95 \% \mathrm{CI})$ & OR & $95 \% \mathrm{CI}$ \\
\hline Age & $-0.002(0.029)$ & 0.938 & $0.017(0.035)$ & 0.618 & 0.998 & $(0.976-1.020)$ & 0.997 & $(0.978-1.016)$ \\
\hline Time after diagnosis & $0.002(0.054)$ & 0.976 & $0.037(0.066)$ & 0.577 & 1.009 & $(0.965-1.055)$ & 0.986 & $(0.952-1.021)$ \\
\hline Years of education & $0.126(0.158)$ & 0.427 & $0.458(0.191)$ & 0.017 & 0.988 & $(0.870-1.122)$ & 0.886 & $(0.802-0.979)$ \\
\hline Job (Ref: no) & $-0.234(0.424)$ & 0.581 & $0.703(0.514)$ & 0.171 & 1.031 & $(0.734-1.449)$ & 0.85 & $(0.647-1.116)$ \\
\hline Smoking (Ref: no) & $-0.047(0.425)$ & 0.912 & $-2.505(0.508)$ & $<0.001$ & 1.062 & $(0.756-1.494)$ & 1.869 & $(1.435-2.435)$ \\
\hline Seeking help (Ref: no) & $0.411(0.593)$ & 0.488 & $-0.974(0.719)$ & 0.176 & 0.972 & $(0.616-1.536)$ & 1.37 & $(0.934-2.009)$ \\
\hline Body mass index & $0.041(0.045)$ & 0.362 & $0.214(0.054)$ & $<0.001$ & 0.998 & $(0.958-1.040)$ & 0.956 & $(0.930-0.982)$ \\
\hline Gleason low (Ref: high) & $-0.058(0.514)$ & 0.911 & $-0.098(0.623)$ & 0.876 & 0.976 & $(0.642-1.483)$ & 1.017 & $(0.733-1.412)$ \\
\hline $\begin{array}{l}\text { Gleason moderate (Ref: } \\
\text { high) }\end{array}$ & $-0.367(0.462)$ & 0.427 & $-0.018(0.561)$ & 0.974 & 0.893 & (0.614-1.299) & 0.99 & $(0.737-1.331)$ \\
\hline Stage 1 (Ref: 3 ) & $2.613(0.556)$ & $<0.001$ & $1.530(0.681)$ & 0.025 & 3.037 & $(1.803-5.114)$ & 0.738 & $(0.508-1.073)$ \\
\hline Stage 2 (Ref: 3 ) & $1.157(0.431)$ & 0.007 & $1.388(0.523)$ & 0.008 & 1.415 & (1.018-1.965) & 0.709 & $(0.539-0.933)$ \\
\hline PEDT score & -- & -- & $0.086(0.042)$ & 0.04 & -- & -- & 0.976 & $(0.955-0.998)$ \\
\hline IIEF-5 score & $0.058(0.028)$ & 0.04 & -- & -- & 1.03 & (1.007-1.053) & -- & -- \\
\hline
\end{tabular}

diagnosis, 674 (63.7\%) had PE and 698 (66.2\%) had ED (Table 1). According to self-reported PEDT score, 749 (71.1\%) suffered from PE using the cutoff $\geq 11$ and 829 (78.7\%) using the cutoff $\geq 9$. According to self-reported IIEF-5 score, 331 (31.4\%) had ED using the cutoff $\leq 7 ; 907$ $(86.1 \%)$ using the cutoff $\leq 22$ (Table 2 ). The prevalence of both PE and ED was similar across different Gleason grades whether the diagnostic method was according to urologists or self-reports. However, the prevalence of PE decreased and that of ED increased according to progressing TNM stages. The trend was much more obvious when using the self-reported PEDT at cutoff $\geq$ 11 and IIEF-5 at cutoff $\leq 22$.

The ability of the PEDT and IIEF to differentiate patients with a sexual problem from healthy ones was demonstrated using ROC curve. Results showed that the AUCs were satisfactory with a score of 0.944 for the PEDT and 0.969 for the IIEF-5. The recommended cutoffs were $\geq 11$ for the PEDT and $\leq 17$ for the IIEF-5 (Table 3 ). The same results were found when the participants were divided into help-seekers $(\mathrm{n}=123)$ and non-help-seekers $(n=931)$.

Multiple linear regression and ordinal logistic regression had similar predictors on PEDT and IIEF5 scores. TNM staging $(\beta=2.613$ and 1.157 for linear regression; $\mathrm{OR}=3.037$ and 1.415 for ordinal regression) and IIEF-5 score $(\beta=0.058 ; \mathrm{OR}=1.030)$ significantly predicted the PEDT scores, while all other factors were nonsignificant. As for IIEF-5 scores, years of education $(\beta=0.458 ; \mathrm{OR}=0.986)$, smoking status $(\beta=-2.505$; $\mathrm{OR}=1.869)$, BMI $(\beta=0.214 ; \mathrm{OR}=0.956)$, TNM staging $(\beta=1.530$ and 1.388; $\mathrm{OR}=0.738$ and 0.709), and PEDT score $(\beta=0.086 ; \mathrm{OR}=0.976)$ were significant predictors (Table 4).

\section{Discussion}

The prevalence of sexual dysfunction diagnosed by urologists in our prostate cancer patients (PE: 63.7\%; ED: $66.2 \%$ ) was higher than that in a general population (PE: 16-38\%; ED: 19-52\%) (Rosen et al., 1999; Spector and Carey, 2009). The high prevalence may be attributable to the common treatments of radiotherapy and radical prostatectomy on prostate cancer patients (Aus et al., 2005; Walz et al., 2008) the rates of ED in prostate cancer patients are as high as $69 \%$ for those underwent radical 
prostatectomy (Dubbelman et al., 2006) and 62\% for those received radiotherapy (Beard et al., 1998). Our results additionally provide the prevalence of sexual problems for prostate cancer patients at different TNM stages. Prostate cancer patients at stage 3 had less PE but more ED problems as compared with those at stages 1 and 2 . However, the reason of different prevalence rates in different stages cannot be explained using our current data and future research is warranted to investigate further. Nevertheless, our results suggested that oncologists may pay more attentions to PE problems on patients at stages 1 and 2, while more to ED problems on patients at stage 3 .

Another important finding of our study is the cutoffs for PE and ED. Although previous studies suggested the valid cutoffs with extremely high accuracy on PE (Symonds et al., 2007) and ED (Rosen et al., 1999), the suggested cutoffs were based on general populations. That is, we cannot make sure that whether the cutoffs are applicable for prostate cancer patients. It seems that using the cutoffs based on general populations to diagnose a sexual problem of a prostate cancer patient is appropriate, and studies(Choo et al., 2010; Ong et al., 2015; Saitz et al., 2013) on prostate cancer patients tended to do so. However, we believed that confirming the cutoffs is essential because prostate cancer patients may have different conditions from general population does in terms of their sexual functions. Our results showed that the best cutoffs are PEDT score $\geq 11$ for a diagnosis of PE and IIEF-5 score $\leq 17$ for a diagnosis of ED. In addition, the sensitivity, 1-specificity, and predictive ability of the cutoffs were very high in our study; thus, we are confident that our proposed cutoffs are useful for oncologists to detect a sexual problem of a prostate cancer patient without further examinations, or consulting an experienced urologist.

Our regression models, including linear regression and ordinal logistic regression, showed that smoking had negative impact on erectile function. This finding is consistent to other studies on different populations, such as those with diabetes mellitus (Bortolotti et al., 2001) and general population (Chew et al., 2009). In addition, the PE problem positively predicted the ED problem and vice versa though the effect was small. Because both PE and ED problems are one kind of sexual problems, they are very likely to be mutually correlated. However, the small effects suggested that PE and ED problems are different parts in sexual problem, and should be separately examined and intervened.

Several study limitations need to be mentioned. First, we did not collect any data on comorbidities which made it impossible to control for the effects of comorbidity on sexual dysfunctions. Second, the diagnosis of PE and an ED - and therefore our base of comparison - depended on the observation of an urologist, and not on an objective measures, such as intravaginal ejaculation latency time (Waldinger et al., 2004) and laboratory evaluations (Bodie et al., 2003). However, the urologists doing the assessment were well-trained Clinicians with many years of experience. Third, our data were cross-sectional, and the casual relationships between potential factors and PE/ ED problems cannot be determined.
In conclusion, the prevalence of sexual problems is high in prostate cancer patients, and both the PEDT and the IIEF-5 are effective measures for oncologists to diagnose sexual problems in prostate cancer patient cohorts. Both measures should be simultaneously administered to comprehensively capture the different aspects of sexual function in prostate cancer patients.

\section{References}

Aus G, Abbou CC, Bolla M, et al (2005). EAU guidelines on prostate cancer. Eur Urol, 48, 546-51.

Beard CJ, Lamb C, Buswell L, et al (1998). Radiation-associated morbidity in patients undergoing small-field external beam irradiation for prostate cancer. Int J Radiat Oncol Biol Phys, 41, 257-62.

Benson CR, Serefoglu EC, Hellstrom WJ (2012). Sexual dysfunction following radical prostatectomy. J Androl, 33, 1143-54.

Bianco FJ, McHone BR, Wagner K, et al (2009). Prevalence of erectile dysfunction in men screened for prostate cancer. Urol, 74, 89-93.

Bodie J, Lewis J, Schow D, et al (2003). Laboratory evaluations of erectile dysfunction: an evidence based approach. J Urol, 169, 2262-4

Bortolotti A, Fedele D, Chatenoud L, et al (2001). Cigarette smoking: a risk factor for erectile dysfunction in diabetics. Eur Urol, 40, 392-6.

Chen RC, Vetter RJ, Lukka H, et al (2014). Recommended patient-reported core set of symptoms to measure in prostate cancer treatment trials. J Natl Cancer Inst, 106, 132.

Choo R, Long J, Gray R, et al (2010). Prospective survey of sexual function among patients with clinically localized prostate cancer referred for definitive radiotherapy and the impact of radiotherapy on sexual function. Support Care Cancer, 18, 715-22.

Chew KK, Bremner A, Stuckey B, et al (2009). Is the relationship between cigarette smoking and male erectile dysfunction independent of cardiovascular disease? Findings from a population-based cross-sectional study. J Sex Med, 6, 222-31 .

Dubbelman YD, Dohle GR, Schröder FH (2006). Sexual function before and after radical retropubic prostatectomy: a system review of prognostic indicators for a successful outcome. Eur Urol, 50, 711-18.

DSM-5: Diagnostic and Statistical Manual of Mental Disorders (5th ed.). Washington (DC): American Psychiatric Association; 2013

Diagnostic and statistical manual of mental disorders, fourth edition, text revision: DSM-IV-TR. Washington (DC): American Psychiatric Association; 2000.

Fan J, Upadhye S, Worster A (2006). Understanding receiver operating characteristic (ROC) curves. CJEM 2006,8,19-20.

Gallo L, Perdona S, Gallo A (2010). The role of short frenulum and the effects of frenulectomy on premature ejaculation. $J$ Sex Med, 7, 1269-76.

Gagnon DD, Polverejan E (2008). Premature Ejaculation: results from a five-country European observational study. Eur Urol, 53, 1048-57.

Giuliano F, Patrick DL, Porst H, et al (2014). Female partner's perception of premature ejaculation and its impact on relationship breakups, relationship quality, and sexual satisfaction. J Sex Med, 11, 2243-55.

Incrocci L, Slob AK, Levendag PC (2002). Sexual (dys)function after radiotherapy for prostate cancer: a review. Int J Radiat Oncol Biol Phys, 52, 681-93.

Ong WL, McLachlan H, Millar JL (2015). Prevalence of baseline 
Chung-Ying Lin et al

erectile dysfunction (ED) in an Australian cohort of men with localized prostate cancer. J Sex Med, 12, 1267-74.

Pakpour AH, Yekaninejad MS, Nikoobakht MR, et al (2014) . Psychometric properties of the Iranian version of the Premature Ejaculation Diagnostic Tool. Sex Med, 2, 31-40.

Pakpour AH, Zeidi IM, Yekaninejad MS, et al (2014). Validation of a translated and culturally adapted Iranian version of the International Index of Erectile Function. J Sex Marital Ther, 40, 541-51.

Rosen RC, Cappelleri JC, Smith MD et al (1999). Development and evaluation of an abridged, 5-item version of the International Index of Erectile Function (IIEF-5) as a diagnostic tool for erectile dysfunction. Int J Impot Res, 11, 319-26.

Saitz TR, Serefoglu EC, Trost LW et al (2013). The pre-treatment prevalence and types of sexual dysfunction among patients diagnosed with prostate cancer. Androl, 1, 859-63.

Spector IP, Carey MP (1990). Incidence and prevalence of sexual dysfunctions: a critical review of the empirical literature. Arch Sex Behav, 19, 389-408.

Singer PA, Tasch ES, Stocking C, et al (1991). Sex or survival: tradeoffs between quality and quantity of life. J Clin Oncol, 9, 328-34.

Symonds T, Perelman MA, Althof S, et al (2007). Development and validation of a premature ejaculation diagnostic tool. Eur Urol, 52, 565-73.

Waldinger MD, Zwinderman AH, Schweitzer DH, et al (2004). Relevance of methodological design for the interpretation of efficacy of drug treatment of premature ejaculation: a systematic review and meta-analysis. Int J Impot Res, 16, 369-81.

Walz J, Perrotte P, Suardi N, et al (2008). Baseline prevalence of erectile dysfunction in a prostate cancer screening population. J Sex Med, 5, 428-35. 